\title{
Newborn neurologic examination
}

\author{
Michele Yang, MD
}

$\mathrm{T}$ his is the first article in a series describing the essentials of the pediatric neurologic examination. The series will address the neurologic examination at different developmental stages from the neonate to the teenage years. The goals of the article are to 1) describe the newborn examination and 2) briefly describe the most common neurologic problems seen in the newborn population.

One of the most dreaded calls for the adult neurology resident is the consult from the neonatal intensive care unit (ICU). Faced with the morass of tubes and monitors underneath which lies a tiny infant, the resident is often paralyzed by the daunting task of trying to perform an examination. As one resident put it, "I could tell he was moving all fours, but that was it." With keen observation and a systematic approach, one can obtain a detailed examination of the newborn. The intent of this article is not to be comprehensive, but to provide a simple approach to the examination and evaluation of the newborn. A summary of the neurological examination is provided in the figure. Further details can be found in the reference articles listed below. ${ }^{1,2}$

By being organized and having the right tools, one can perform as comprehensive an examination as in an adult. Tools for examination of the newborn are as follows: 1) bell, 2) ophthalmoscope, 3) reflex hammer, 4) cotton-tipped application, 5) measuring tape.

Before proceeding to a discus- sion of the neurologic examination, two important aspects of the general physical examination should be noted. Keeping in mind that the neurologic system is derived from ectoderm, one should pay particular attention to the examination of the skin. Outgrowths such as encephaloceles, cutaneous lesions such as port-wine stains, and the presence of sacral dimples or sinuses should be sought as clues to underlying neurologic dysfunction. Additionally, head circumference should be measured with a tape measure. The normal term infant's head circumference is $35 \mathrm{~cm} \pm 2 \mathrm{~cm}$ and is a reflection of the underlying intracranial volume. Thus, it is a good way to monitor for intracranial masses and increased intracranial pressure. Additionally, macrocephaly and microcephaly can be indications of underlying metabolic, genetic, or infectious processes.

\section{Neurologic examination \\ Mental status}

One of the best times to examine a baby is between feeds. If interrupted during a feed, the baby may cry excessively, limiting the examination, and if examined immediately after a feed, the baby may be too sleepy to obtain an optimal examination. Observation of the newborn's spontaneous eye opening, movements of the face and extremities, and response to stimulation are essential for the mental status examination. Arousal is defined by the duration of eye opening and spontaneous movement of the face and extremities. Before 28 weeks gestation, the newborn states of wakefulness and sleep are difficult to distinguish. As the newborn matures, however, there is increasing duration, frequency, and quality of alertness. Again, it is important to keep in mind that these states will depend on the patient's last feed and activity (such as placement of an IV). An irritable infant is one who is agitated and cries with minimal stimulation and is unable to be soothed. Lethargic infants cannot maintain an alert state.

\section{Cranial nerves}

Cranial nerves (CN) II and III can be tested by the pupillary reflex, which appears consistently at 32 to 35 weeks gestation. A 28week infant will blink to light shone into the eyes, testing CN II and VII. Beginning at 34 weeks of gestation, an infant will be able to fix and follow on an object, thus testing CN II, III, IV, and VI. Spontaneous roving eye movements are common at 32 weeks gestation, as are dysconjugate eye movements in the term infant when not fixing on an object. Another maneuver to test eye movements is the following: hold the baby underneath the axilla and spin the baby from side to side to test the oculovestibular reflex. Not only does this test acuity in the duration of the postrotational nystagmus, but this also tests the integrity of the vestibular system. Facial sensation $(\mathrm{CN} \mathrm{V})$ is tested with pinprick and by observing facial grimace or change in sucking. Facial symmetry and movement should be observed in both the quiet state and during active move- 


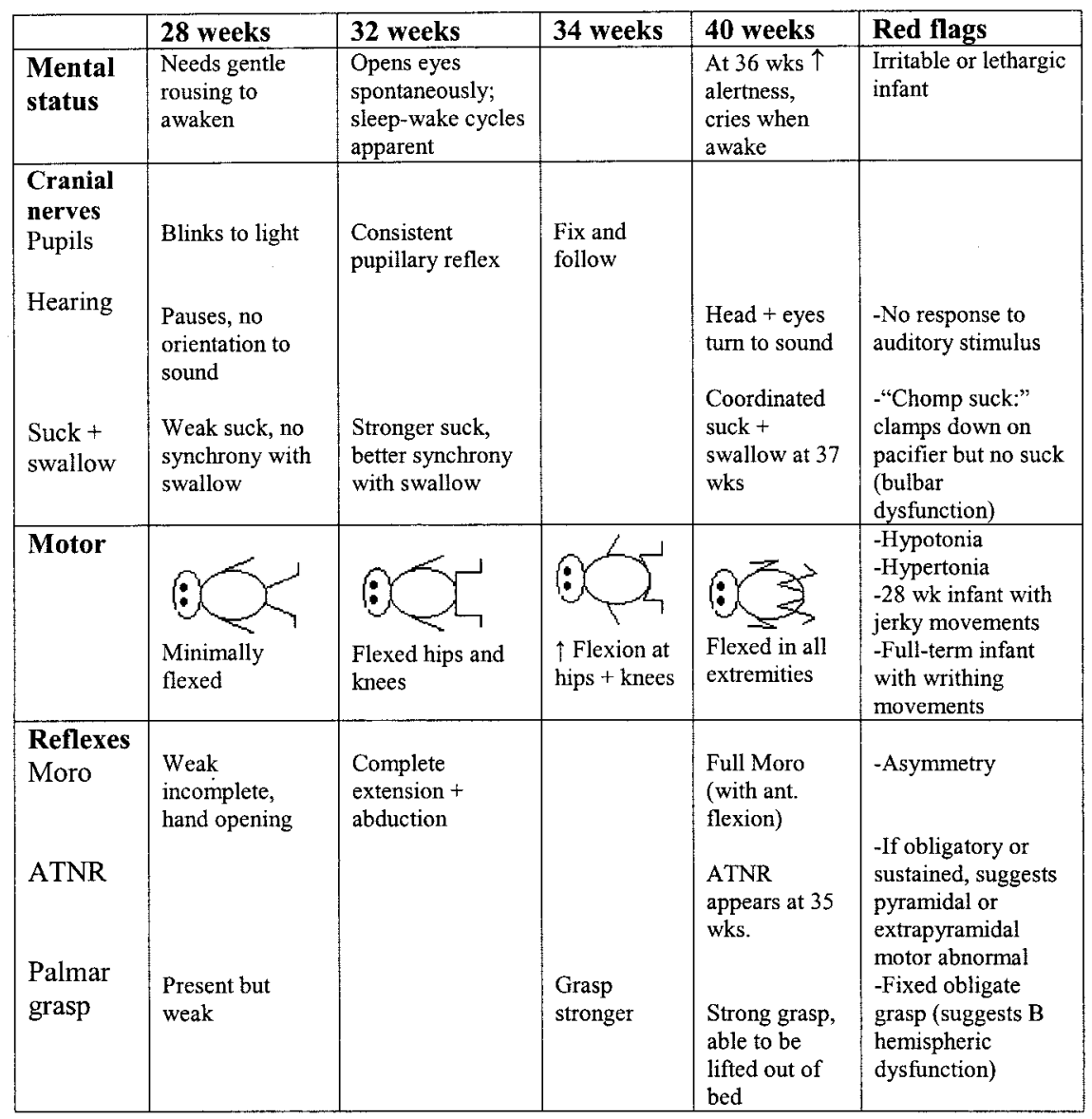

ment (such as crying). Hearing (CN VIII) can be tested with a bell, keeping in mind that a ringing bell within an isolette can be quite loud and generate $90 \mathrm{~dB}$. The newborn may have a very subtle response to auditory stimulus and respond with only a blink. To test CN V, VII, and XII, the newborn can be observed sucking on a pacifier. This can also be used to evaluate CN IX and X, which are tested when the baby swallows. The 28-week infant can suck and swallow but the synchrony of breathing and feeding is not well developed. As the brainstem matures, coordination improves by the 32 nd to 34 th week. Palpation of the sternocleidomastoid (CN XI) may be difficult in the newborn, but may be facilitated by extending the head on the side of the bed with the infant in a supine position. Now the bulk of the muscle can be palpated as the head is turned to the side.

\section{Motor examination}

Observation of the resting posture can reveal the symmetry and maturity of the passive tone. It is important to keep the head midline to avoid asymmetries in tone related to the asymmetric tonic neck reflex. Flexor tone tends to develop first in the lower extremities and proceed cephalad. A 28 -week infant will lie with minimally flexed limbs and have minimal resistance to passive movement of all extremities. In contrast, at 32 weeks, the newborn develops flexor tone at the hips and knees, with some resistance to manipulation of the lower extremities. This progression correlates with increasing myelination of the subcortical motor pathways originating in the brainstem. By 36 weeks, the infant develops flexion at the elbows, and by term, the infant is flexed in all extremities. The quality of the infant's movements develops as well. For example, the 28-week infant will have writhing movements of the extremities, but by term the movements are best described as large amplitude "swatting" movements. A 28-week infant
Figure. Summary of the neurologic examination with respect to gestational age. ATNR = asymmetric tonic neck reflex. with jerky movements is abnormal and drug withdrawal should be suspected. Conversely, a term infant with choreoathetoid movements should be evaluated for a number of potential structural or metabolic abnormalities.

\section{Sensory examination}

In the newborn, the examination is limited to touch and pinprick. Particular emphasis should be placed on dermatomal evaluation of the lower extremities, especially in the sacral region in a child with a neural tube defect. Assessment of sensation can be made by using the sharp end of a cotton applicator on the face and observing the facial grimace or change in state of the infant.

\section{Reflexes}

Reflexes can be easily elicited in the biceps, brachioradialis, knees, and ankles. Cross adductor responses and unsustained clonus are not uncommon in the newborn. Many child neurologists agree that the plantar response is not helpful, 
as many factors may elicit flexor or extensor responses inadvertently.

\section{Primitive reflexes}

Of the primitive reflexes that can be elicited in the newborn, the following are the most important to perform.

A full Moro reflex consists of bilateral hand opening with upper extremity extension and abduction, followed by anterior flexion of the upper extremities, then an audible cry. This is best elicited by dropping the head in relation to the body, into the examiner's hands.

The asymmetric tonic neck reflex is elicited by rotating the head to one side, with subsequent elbow extension to the side the head is turned and elbow flexion on the side of the occiput.

The palmar grasp reflex is elicited by stimulating the palm with an object. The palmar grasp is present at 28 weeks gestation, strong at 32 weeks, and is strong enough at 37 weeks gestation to lift the baby off the bed. This reflex disappears at 2 months of age with the development of a voluntary grasp.

To test the placing reflex, the infant is held under the axilla in an upright position, and the dorsal aspect of the foot is brushed against a tabletop. The infant's hip and knee will flex, and the infant will appear to take a step. This reflex is useful if asymmetry occurs and may indicate a lesion in the basal ganglia, brainstem, or spinal cord. However, performing this reflex can be limited by the constraints of the isolette, endotracheal tube, or multiple lines.

\section{Patterns of neurologic dysfunction}

\section{Parasagittal cerebral injury}

With diffuse decreased cerebral perfusion in asphyxia, ischemic changes occur in the arterial border zones of the cerebral hemispheres. As a consequence, the neonate will exhibit hypotonia of the upper extremities, with weakness particularly in the shoulders, in addition to lower extremity weakness.

Periventricular leukomalacia and paraventricular/intraventricular hemorrhage

The germinal matrix is a vascular-rich zone containing pluripotential cells from which the cortex develops. It is susceptible to bleeding in the preterm infant. With abnormalities in cerebral perfusion, these areas hemorrhage and often develop infarction in the deep white matter of the hemispheres. As a result, these babies can develop initial weakness in their lower extremities, although often they may have relatively normal examinations. As myelination progresses in the corticospinal tracts with maturation, however, the infants can develop increased lower extremity tone and increased reflexes by about 4 to 5 months of age. This is in contrast to parasagittal cerebral injury where both upper and lower extremities are involved.

Spinal cord

Traumatic cord lesions can develop in infants, especially in the setting of a difficult breech delivery with a tear in the cervical dura. This results in symmetric lower extremity paralysis with sparing of the face and cranial nerves and involvement of the sphincters.

\section{Peripheral nerve}

The most common injury involving the peripheral nerve is the proximal cervical roots $\mathrm{C} 5, \mathrm{C} 6$, and $\mathrm{C} 7$, usually in the setting of a traumatic delivery with shoulder dystocia. In Erb's palsy there is paralysis of shoulder abduction, elbow flexion, and finger extension, so that the arm is held extended, externally rotated with flexion at the wrist. No biceps reflex can be elicited although one may be present in the triceps. Sensation is diminished in the lateral aspect of the arm.
Neuromuscular junction and muscle

In myasthenia gravis and infantile botulism, diffuse hypotonia and weakness are present, often in conjunction with $\mathrm{CN}$ involvement. In congenital myopathies, proximal extremity weakness is prominent and can be marked by limb deformities if the onset occurred in utero.

Seizures vs jitteriness

A common consult from the neonatal ICU is for seizures. In a newborn, many movements such as sucking may be mistaken for seizure; on the other hand, subtle movements such as bicycling of the legs may be overlooked as a manifestation of seizure. A good rule of thumb is to obtain an EEG to determine if seizure activity is present. Jitteriness may be difficult to distinguish from seizure, but a few clinical clues may help. Jitteriness from drug withdrawal often presents with tremors, whereas clonic activity is most prominent in seizures. Jitteriness tends to be stimulus-sensitive, becoming most prominent after startle, and its activity can cease by holding onto the baby's arm, neither of which is true in seizures. Additionally, seizures tend to be accompanied by autonomic changes as well.

A detailed neurologic examination can be accomplished through a systematic approach and close observation of the newborn, keeping in mind that the examination changes with gestational age. A careful examination can thus guide the examiner in choosing the most appropriate diagnostic tests.

\section{References}

1. Volpe JJ. Neurological evaluation. In: Volpe JJ. Neurology of the newborn. 4th edition. Philadelphia: W.B. Saunders, 2001;103-133.

2. Painter MJ. Neurological evaluation of newborns, infants, and older children. In: Albright AL, Pollack IF, Adelson PD. Principles and practice of pediatric neurosurgery. New York: Thieme, 1999;3-19. 


\title{
Neurology
}

\author{
Newborn neurologic examination \\ Michele Yang \\ Neurology 2004;62;E15-E17 \\ DOI 10.1212/WNL.62.7.E15
}

This information is current as of April 12, 2004

\section{Updated Information \&}

Services

Permissions \& Licensing

Reprints including high resolution figures, can be found at:

http://n.neurology.org/content/62/7/E15.full

Information about reproducing this article in parts (figures,tables) or in its entirety can be found online at:

http://www.neurology.org/about/about_the_journal\#permissions

Information about ordering reprints can be found online:

http://n.neurology.org/subscribers/advertise

Neurology ${ }^{\circledR}$ is the official journal of the American Academy of Neurology. Published continuously since 1951, it is now a weekly with 48 issues per year. Copyright . All rights reserved. Print ISSN: 0028-3878. Online ISSN: 1526-632X.

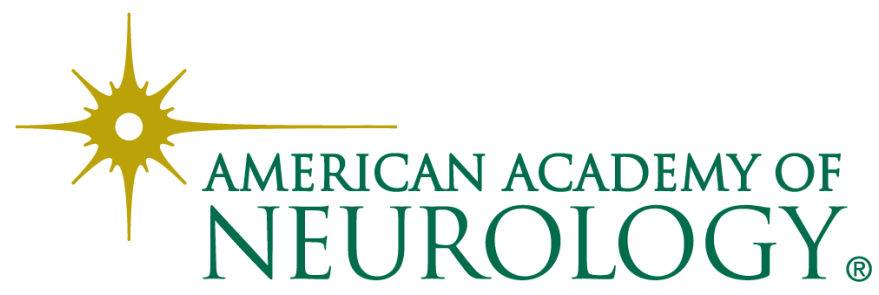

